\title{
Transdermal hormone therapy and bone health
}

\author{
Lee P Shulman \\ Division of Reproductive Genetics, \\ Department of Obstetrics and \\ Gynecology, Feinberg School of \\ Medicine of Northwestern University, \\ Chicago, Illinois, USA
}

\begin{abstract}
The clinical aftermath of the reporting of the initial findings of the Women's Health Initiative (WHI) in 2002 was a profound reduction in the use of hormone therapies by menopausal women. This reduction led to a well documented increase in vasomotor symptoms and vaginal atrophy among those women who discontinued their hormone regimens. However, another adverse impact among these women, as well as many other menopausal women, is the well recognized increased likelihood of osteoporosis resulting from the decline in circulating estradiol levels associated with natural and surgical menopause. Although the use of non-hormonal drugs such as bisphosphonates has been shown to reduce the risk of fracture in women with osteoporosis, bisphosphonates have not been shown to reduce the risk of fracture in non-osteoporotic women. Indeed, only oral estrogen (as demonstrated in the WHI studies) has been shown to reduce the risk of fracture in osteoporotic and non-osteoporotic women. As non-oral hormone therapies have been shown to be as effective in treating vasomotor symptoms and vulvovaginal atrophy and to have a different (and perhaps more beneficial) physiological effect than oral regimens, it behooves us to assess the impact of non-oral hormone regimens on bone mineral density and fracture risk. Although there are no clinical trials that primarily assess the impact of non-oral regimens on fracture risk in menopausal women, numerous studies are consistent in demonstrating the positive impact of non-oral regimens in maintaining and increasing bone mineral density among users, even for those women using estrogen doses that are considered to be "too low" to have a beneficial impact on other menopausal symptoms.
\end{abstract}

Keywords: menopause, hormone, estrogen, non-oral, bone, osteoporosis, fracture

\section{Introduction}

There is almost universal agreement that hormone therapy (HT) effectively treats and prevents menopausal symptoms and osteoporosis. These effects have been reported in a variety of well-respected observational and randomized clinical trials (RCTs) (Raz and Stamm 1993; Writing Group for the Pepi Trial 1996; Greendale et al 1998; Notelovitz et al 2000). Controversial issues concerning additional potential risks and benefits of HT have received much attention in the medical literature and the lay press, particularly as result of the reports from the Women's Health Initiative (WHI). Indeed, the reports from the WHI have cast doubt concerning the safety of all menopausal hormone therapies and caused fear and concern among laity as result of the extensive press coverage of the ongoing findings of this trial.

Although the WHI is by far the largest study of the clinical impact of oral hormone therapy in menopausal women, it is profoundly limited in that it has evaluated only two oral hormonal regimens and its protocol is mostly limited to the use of hormone therapy in an older, but perhaps more importantly, a mostly asymptomatic cohort of women who would not have been optimal candidates for hormone therapy even during the performance of the study. In particular, the WHI evaluated no nonoral menopausal hormone therapies; however, regulatory agencies and others have unfortunately considered the results of the WHI to be applicable to all regimens until data are presented that counter the safety findings of the WHI. Indeed, this sweeping 
generalization ignores the considerable evidence, even from the WHI, that demonstrates that factors such as age and health of the patient (Manson et al 2007) and method of delivery of the sex steroids (Scarabin et al 2003) play seminal roles in determining the overall safety of the hormone therapy.

With regard to transdermal delivery of estrogen and progestin for hormone therapy, considerable information exists that demonstrates that while the efficacy of transdermal systems for the relief of menopause-related symptoms and conditions is comparable to oral regimens, the physiologic effect of these delivery systems is considerably different from that observed with oral regimens (Scarabin et al 2003). To this end, the use of all estrogen-based therapies have been shown to consistently reduce vasomotor symptoms, prevent and treat vulvo-vaginal atrophy and prevent bone mineral density loss in the vast majority of menopausal women regardless of the cause of menopause. This review will assess the role of transdermal hormone delivery systems for the prevention and treatment of post-menopausal osteoporosis.

\section{Post-menopausal osteoporosis}

Osteoporosis is a significant cause of morbidity and mortality in post-menopausal women. At the age of 50, a woman has a $40 \%$ lifetime chance of an osteoporotic fracture and a $16 \%$ chance of a hip fracture (Chrischilles et al 1991). Of greatest concern is that individuals who suffer from osteoporotic hip fractures have up to a $24 \%$ risk of death in the year following the hip fracture (Johnell and Kanis 2006). These increased risks for fracture and their associated morbidity and mortality are directly attributable to the loss of bone mineral density resulting from the loss of physiologic levels of estrogen resulting from surgical or natural menopause.

After menopause, low estrogen levels lead to an imbalance in bone metabolism and a higher rate of bone resorption. This process leads to a steady decrease in bone mineral density (BMD) and bone strength and can result in spinal, hip or other bone fractures in the absence of traumatic events. While administration of calcium, vitamin D, and exercise may temporarily retard bone resorption, they do not prevent osteoporosis or fractures.

\section{Osteoporosis therapies}

Bisphosphonates and raloxifene have demonstrated fracture prevention in women with osteoporosis. However, no data show effectiveness in fracture reduction among women without osteoporosis, thus causing concern to those women looking for fracture reduction before the onset of osteoporosis (Shulman 2004). To this end, the hormone therapy arms (combination therapy [HT] and estrogen - alone therapy [ET]) of the WHI showed significant reductions in hip fracture in a population not previously diagnosed with osteoporosis (Cauley et al 2003). At the end of year 3, BMD had increased by $3.7 \%$ in the HT group and $0.14 \%$ in the placebo group $(\mathrm{p}<0.001)$. At the end of 5.6 years, $8.6 \%(n=733)$ of patients receiving HT experienced a fracture, compared with $11 \%(\mathrm{n}=896)$ in the placebo group. Cumulatively, HT reduced the risk of fracture by $33 \%$ in a population already at risk for fracture: $44 \%$ of the WHI subjects were older than 65 years of age. Similar findings were demonstrated in comparing the ET group with the placebo group.

\section{Transdermal hormone therapies}

Oral estrogen use is strongly associated with maintenance and increase in bone mineral density and a reduction in fracture risk; however, does transdermal delivery of estrogen, with or without a progestin, provide a similar salutary effect on bone mineral density and fracture risk? Transdermal estrogen therapies have long been shown to be effective in maintaining or increasing bone mineral density among menopausal women (Samsioe 2004). However, the study by Ettinger and colleagues (2004) showing beneficial bone mineral density effects of an "ultralow-dose" transdermal estradiol patch provides important information to women and clinicians considering osteoporotic preventive and therapeutic options. Ettinger and colleagues were able to show in this randomized, placebo-controlled trial that a transdermal patch delivering $0.014 \mathrm{mg} / \mathrm{d}$, did not impact vasomotor symptomatology but did increase lumbar spine bone mineral density by $2.6 \%$ versus 0.6 in the placebo group $(\mathrm{p}<0.001)$ and did increase total hip bone mineral density by $0.4 \%$ compared to a $0.8 \%$ reduction among women in the placebo group $(p<0.001)$. These findings lead to the eventual approval of this method (Menostar ${ }^{\mathrm{TM}}$ ) for the prevention of bone mineral density loss in menopausal women, although the concomitant finding of one woman in the estradiol group (and no women in the placebo group) developing endometrial hyperplasia probably influenced the FDA to include a somewhat restrictive and complicated recommended clinical algorithm for the use of this transdermal regimen in the absence of a progestational agent. Indeed, studies of low and ultralow-dose regimens in all menopausal age groups have shown a consistent and significant increase in bone mineral density and reductions in bone turnover markers and no increased risk of endometrial hyperplasia or other adverse events compared to oral regimens (Richman et al 2006). 
If an ultralow-dose transdermal therapy is effective in maintaining, or even increasing, bone mineral density, what about the bone impact of a combination transdermal therapy using a higher estrogen dose? Warming and colleagues (2005) studied a combination patch with delivering a daily dose of $45 \mathrm{mcg} 17 \beta$-estradiol and either 30 or $40 \mathrm{mcg}$ levonorgestrel as a 7-day patch over a 2-year study period among osteopenic, but not osteoporotic, postmenopausal women. Bone mineral density measurements at the lumbar spine, hip and total body increased by 8,6 and $3 \%(\mathrm{p}<0.001)$ respectively, in the hormone groups compared to placebo. All bone markers except for urinary Calcium ( $\mathrm{uCa}$ ) were significantly reduced in the hormone groups compared to the placebo group; $\mathrm{uCa}$ was unchanged. In addition, no significant dose-related effect of levonorgestrel was found and no cases of endometrial hyperplasia, uterine cancer or breast cancer were reported during the study period. As with other estrogen-alone and combination regimens, this study provides conclusive evidence of the benefit of transdermal estrogen therapy for the prevention of menopause-related osteoporosis.

Unfortunately, there are no meaningful studies directly evaluating fracture risk in women using transdermal hormone therapies for menopause management. However, the FDA and others have concluded that bone mineral density assessment by bone densitometry protocols is an accurate surrogate marker for predicting fracture risk in menopausal women. Nonetheless, a question concerning hormonal therapies is raised and continues to be an integral part of ongoing research; that is, which delivery system, oral or transdermal, is better? Again, transdermal delivery systems clearly are associated with a different physiological impact than oral regimens (Shulman 2004) which may provide a slight benefit for the use of such regimens given the comparable beneficial clinical outcomes of oral and non-oral regimens. The potential of these pharmacokinetic differences having a novel clinical impact on users is best delineated by the study by Scarabin and colleagues (2003) who showed that women using transdermal hormone therapies had a risk of VTE comparable to non-users of hormone therapy and those who used oral therapies had an estimated risk of 4.0 compared with women using transdermal regimens. Other differences found with transdermal use compared to oral use include a greater reduction in sympathetic tone (Girdler et al 2004), little to no increases in C-reactive protein and an overall reduced impact on risk factors for atherosclerotic vascular disease (Ho et al 2006).

Given the physiologic differences resulting from oral and non-oral hormone regimens, is there any evidence of differences comparing oral and non-oral regimens concerning bone health? For the most part, clinical impact is considered to be a dose-related phenomenon whether it be relief of vasomotor symptoms or increases in bone mineral density. Indeed, a head-to-head comparison would be almost impossible as there would be no good clinical approach to determining the relative "sameness" of an oral and transdermal dose of estrogen and progestin. Nonetheless, a recent study by Yasui and colleagues showed that the effect of transdermal estrogen was weaker than that of oral estrogen (conjugated equine estrogen) on undercarboxylated osteocalcin (ucOC), a sensitive marker of vitamin $\mathrm{K}$ status. ucOC is increased after natural or surgical menopause; hormone therapy is associated with reduced levels of ucOC. The stronger effect of oral hormone therapy in this study was believed to be associated with the higher triglyceride levels associated with oral therapy, a physiologic effect not considered to be generally salutary. Regardless, this study gives us an interesting perspective on the different physiologic effects of oral and non-oral hormone therapies on bone, despite apparently similar beneficial clinical outcomes accrued by both delivery systems.

Perhaps the greatest drawback of any hormone therapy is compliance and persistence of use. The poor persistence with these therapies is not surprising given the preventive nature of their use. Ettinger and Pressman (1999) showed that 2 of 3 women who begin hormone therapy discontinue within a year, and continuation with transdermal hormone therapy is not as good as with oral regimens. To this end, clinicians should provide comprehensive counseling concerning menopause and hormone therapy to women who are considering using hormone therapy and empower those women who choose to use hormone therapy to select a regimen that is most likely to be well incorporated into their lifestyle and, if situations change with the use of a particular regimen, be ready to change the specific hormone therapy so as to encourage its use for as long as the woman remains an appropriate candidate for postmenopausal hormone therapy.

\section{Conclusions}

The benefits of estrogen therapy for postmenopausal women for preventing bone mineral density loss are clear and unequivocal. Oral and non-oral therapies have been shown to increase bone mineral density in surgically and naturally menopausal women. Indeed, a reduction in bone fracture has been shown to accrue to women who are osteopenic and who use oral estrogen therapy. Despite the lack of fracture data for women using transdermal therapies, it is clear that these therapies result in a similar salutary impact on bone 
as observed with oral regimens. Even studies evaluating an ultralow, or subtherapeutic dose of transdermal hormone therapy have shown important bone effects with regard to maintenance and small increases in bone mineral density among users.

What appears to be the major issue that currently impacts the use of hormone therapy is the definition of "symptomatic menopause." In the United States, Europe and elsewhere, many clinicians reserve the use of systemic hormone therapies for only those women with moderate to severe vasomotor symptomatology. This practice has arisen out of the initial interpretation of the WHI studies and the ongoing misperceptions of hormone use among women. While I do not believe that hormone use is for every menopausal woman, or that the use of hormone therapy is not associated with clear, and in some cases, severe adverse events, the positive impact of hormone therapy on bone along with the profound morbidity and mortality of osteoporosis must be considered within the clinical paradigm of hormone therapy use in menopause. Up until now, the benefits of hormone therapy have been, at best, a secondary benefit accrued by those women with symptoms severe enough to warrant consideration of systemic therapy. However, the absence of benefit of bisphosphonates for non-osteoporotic women along with the benefits and unique physiologic impact of non-oral regimens, including ultralow-dose regimens, should give consideration of the use of particular estrogen regimens for the prevention of osteoporosis in selected women who are not suffering from severe menopause-associated symptoms.

\section{References}

Cauley JA, Robbins J, Chen Z, et al. 2003. Effects of estrogen plus progestin on risk of fracture and bone mineral density. JAMA, 290:1729-38.

Chrischilles EA, Butler CD, Davis CS, et al. 1991. A model of lifetime osteoporosis impact. Arch Intern Med, 151:2026-32.

Ettinger B, Ensrud KE, Wallace R, et al. 2004. Effects of ultralow-dose transdermal estradiol on bone mineral density: a randomized clinical trial. Obstet Gynecol, 104:443-51.

Ettinger B, Pressman A. 1999. Continuation of postmenopausal hormone replacement therapy in large health maintenance organization: transdermal matrix patch versus oral estrogen therapy. Am J Manag Care, 5:779-85.

Girdler SS, Hinderliter AL, Wells EC, et al. 2004. Transdermal versus oral estrogen therapy in postmenopausal smokers: hemodynamic and endothelial effects. Obstet Gynecol, 103:169-80.

Greendale GA, Reboussin BA, Hogan P, et al. 1998. Symptom relief and side effects of postmenopausal hormones: results from the Postmenopausal Estrogen/Progestin Interventions Trial. Obstet Gynecol, 92:982-8.

Ho JY, Chen MJ, Sheu WH, et al. 2006. Differential effects of oral conjugated equine estrogen and transdermal estrogen on atherosclerotic vascular disease risk markers and endothelial function in healthy postmenopausal women. Hum Reprod, 21:2715-20.

Johnell O, Kanis JA. 2006. An estimate of the worldwide prevalence and disability associated with osteoporotic fractures. Osteoporos Int, $17: 1726-33$.
Manson JE, Allison MA, Rossouw JE, et al. 2007. Estrogen therapy and coronary-artery calcification. New Engl J Med, 356:2591-602.

Notelovitz M, Cassel D, Hille D, et al. 2000. Efficacy of continuous sequential transdermal estradiol and norethindrone acetate in relieving vasomotor symptoms associated with menopause. Am J Obstet Gynecol, 182:7-12.

Raz R, Stamm WE. 1993. A controlled trial of intravaginal estriol in postmenopausal women with recurrent urinary tract infections. $N$ Engl $J$ Med, 329:753-6.

Richman S, Edusa V, Fadiel A, et al. 2006. Low-dose estrogen therapy for prevention of osteoporosis: working our way back to monotherapy. Menopause, 13:148-55.

Samsioe G. 2004. Transdermal hormone therapy: gels and patches. Climacteric, 7:347-56.

Scarabin P-Y, Oger E, Plu-Bureau G, et al. 2003. Differential association of oral and transdermal oestrogen-replacement therapy with venous thromboembolism risk. Lancet, 362:428-32.

Shulman LP. 2004. The menopausal transition: how does route of delivery affect the risk/benefit ratio of hormone therapy? J Fam Pract, Jul Suppl:S13-17.

Scarabin PY, Oger E, Plu-Bureau G, et al. 2003. Differential association of oral and transdermal oestrogen-replacement therapy with venous thromboembolism risk. Lancet, 362:428-32.

Warming L, Ravn P, Christiansen C. 2005. Levonorgestrel and 17 $\beta$-estradiol given transdermally for the prevention of postmenopausal osteoporosis. Maturitas, 50:78-85.

Writing Group for the Pepi Trial. 1996. Effects of hormone therapy on bone mineral density: results from the postmenopausal estrogen/progestin interventions (PEPI) trial. JAMA, 276:1389-96. 